\title{
About the Relevance of Mathematical Programming and Stochastic Optimisation Methods : Application to Optimal Batch Plant Design Problems
}

\author{
Ponsich Antonin*, Azzaro-Pantel Catherine, Domenech Serge, Pibouleau Luc \\ Laboratoire de Génie Chimique UMR 5503 CNRS/INP/UPS \\ 5 rue Paulin Talabot BP1301 \\ 31106 TOULOUSE Cedex 1
}

\begin{abstract}
Due to their large variety of applications, combinatorial optimisation problems led to a great effort to develop efficient solution techniques. But among the diversity of those optimisation methods, the choice of the relevant technique to the treatment of a given problem keeps being hard.

Within the Process Engineering framework, Optimal Batch Plant Design problems provide a good support to tackle, on the one hand, two Mathematical Programming techniques - DICOPT and SBB, implemented in the GAMs software - and, on the other hand, one stochastic method, i.e. a Genetic Algorithm. Various instances of a problem, with an increasing complexity, were solved with these techniques which performances were compared : the results enable to judge their efficiency in order to propose the most appropriate method according to the studied problem instance.
\end{abstract}

Keywords: optimisation methods, genetic algorithm, GAMs, batch plant design.

\section{Introduction}

A wide range of applications drawn from the Process System Engineering area can be formulated as optimisation problems, that often prove to be highly combinatorial. This range of applications covers the simple travelling man problem as well as graph colouring or frequencies allocation for radio-mobile networks. Chemical Engineering also provides combinatorial optimisation examples, for instance reactors or heat exchangers networks, batch plant design. Consequently, a great number of solution techniques were implemented, but it is quite difficult to judge their efficiency a priori : it just appears that a method adapted to a particular example will give better results than a systematic resolution scheme. The objective of this paper is to propose some guidelines which may be useful for the choice of an adequate optimisation technique. This paper is divided into seven sections. The general aims and the used methodology are developed in Section 2. Sections 3 and 4 describe the investigated optimisation methods. Section 5 explains the problem formulation and the studied examples. Some

*Author to whom correspondence should be addressed: Antonin.Ponsich@ensiacet.fr 
typical results are analysed in Section 6. Finally, conclusions and perspectives are presented in Section 7.

\section{Aims and methodology}

A large variety of applications can be formulated as combinatorial optimisation problems. Therefore, important research efforts have been made to propose various solution techniques.

\subsection{A great diversity of optimisation methods}

Among the diversity of these techniques, two important classes can be distinguished. The former is based on deterministic methods. They involve the verification of mathematical properties of the objective function and constraints to ensure a local optimum. But it is well-known that they might be strongly penalized by the studied problem size. The latter is composed of stochastic methods : they only evaluate the objective function at different points of the search space. These points are chosen by using a set of heuristics combined with generations of random numbers. This latter class is characterized by an adaptability to a large range of problems.

Actually, various works propose well-fitted methods that give good results for particular cases but there still exists a deficiency of studies that would evaluate method performance and justify their use a priori. In this perspective, an Optimal Batch Plant Design problem (OBPD) was solved successively by different approaches : a heuristics way (Modi and Karimi, 1989), a simulated annealing (Patel et al., 1991), a Genetic Algorithm and an ant colony procedure (Wang et al, 1996 and 2002). Recent works (and among them the present study) have shown that an optimal solution better than all the previous ones can be determined with a standard Mathematical Programming technique.

\subsection{Resolution methodology}

So, the aim of this work is to evaluate the behaviour of techniques derived from deterministic and stochastic classes. This paper presents the solution of various examples by two deterministic methods and by a stochastic technique. The set of examples covers a range of problems showing increasing size and complexity. The deterministic methods are based on Mathematical Programming (MP) solvers implemented in the GAMs environment (Brooke et al., 1998). A Genetic Algorithm developed in a previous work (Pibouleau et al., 2004) illustrates the stochastic methods family. This technique will require an additional study on constraint handling, which constitutes a critical point for the application of such techniques. The comparison of the results obtained by each method enables to evaluate their performances and to judge the relevance of their use according to the studied instance of the problem. This study will then stand as a basis for general guidelines helping to choose an appropriate method.

\section{Mathematical Programming methods}

Mathematical Programming techniques were adopted in the study to represent deterministic methods since they are widely used and available in standard optimisation libraries and in modelling environments such as DICOPT and SBB in the GAMs environment (Brooke et al., 1998). 


\subsection{DICOPT}

The DICOPT solver relies on the Outer Approximation algorithm developed by Duran and Grossmann (1986) and was improved several times. It operates by decoupling the problem into one continuous NLP sub-problem and one MILP master-problem, the discrete and continuous variables being successively fixed. The result of the subproblem provides - by means of an NLP solver, here CONOPT3 - an upper bound, if a feasible solution does exist. Then, the problem is relaxed by linearizing the constraints involving binary variables : the resulting discrete master-problem is solved by the MILP solver, i.e. CPLEX : the solution is a lower bound to the global problem. The algorithm terminates when the actual decreasing upper bound intersects the actual increasing lower bound, except when non-convexities lead to sub-optimal NLP solution or cause a cut in the feasible space that eliminates the global optimum.

\subsection{SBB}

The SBB solver is a classical Branch and Bound algorithm, a constructive technique working by relaxing the integrality constraints on the discrete variables. The associated continuous non-linear problems are then solved - with CONOPT3 - in order to reduce the bounds of one relaxed discrete variable and create two others NLP sub-problems. An increasing lower bound is produced by solution of the relaxed NLP sub-problems, while a decreasing upper bound is actualised every time a feasible solution is found. Their intersection is the termination criterion. The key-points of its implementation are the choices of the next variable to be branched on and of the next NLP sub-problem to solve. The number of created NLP sub-problems may seem quite restrictive, but they finally just differ one from another by the variables bounds.

\section{A stochastic method : Genetic Algorithms}

Genetic Algorithms were chosen to illustrate the stochastic methods class since they have proven to be efficient for treating OBPD problems. They also manage a population of individuals, and so are able to provide a set of good solutions when the algorithm ends.

\subsection{General principles}

The principles of GAs just lie on the analogy made between a population of individuals and a set of an optimisation problem solutions. Just like the former, the latter evolves towards a good quality, or adaptation, according to the mechanisms implemented in the GAs. These mechanisms mimic the rules of natural selection stated by Darwin : survival of the best individuals, crossover or mutation. This heuristics set is mixed with a strongly stochastic aspect, leading to the compromise between exploration and intensification in the search space, which makes the GAs efficiency. The GA presented in this study, adapted from Pibouleau et al.(2004), uses quite common genetic operators.

\subsection{Constraint handling}

Since constraints cannot be easily implemented just by additional equations as in MP techniques, their handling is a key-point of GAs. It would seem natural to lay down the limits of the feasible space by eliminating all the solutions violating any constraint. But the more severe these constraints are, the more difficult it is to randomly find one 
feasible solution : as an initial population of feasible solutions must be generated, the effect of this technique on the computational time is strongly penalizing.

So, other ways for constraint handling have to be tackled and tested: (i) elimination as a reference, (ii) penalization of the feasible individuals, (iii) relaxation of the discrete variables range, (iv) tournament involving various numbers of competitors and winners, (v) pseudo-domination method (one tournament for each selection step). The two first techniques are classical and do not need to be related. The third technique is just an enlargement of the feasible space : the minimization will anyway makes the variables to tend within their bounds. The two last methods rely on domination rules stated in different papers (particularly Coello and Mezura, 2002). Basically, they say that :

- a feasible individual dominates an infeasible one ;

- if two individuals are feasible, the one with the best objective function wins ;

- if two individuals are infeasible, the one with the smallest constraint violation wins.

By lack of place, exhaustive results cannot be presented here. The results analysis first underlines the efficiency of the tournament and pseudo-domination methods. Finally, from the study of the evolution of both feasible individuals number and fitness average, the pseudo-domination technique turns out to be slightly more performing and was finally adopted for the more complex examples. The elimination technique was adopted for the smallest ones, since the CPU time is then not so important.

\section{Optimal Batch Plant Design problems}

Within the Process Engineering framework, the Optimal Batch Plant Design (OBPD) problems have been modelled and solved with a lot of approaches yet.

\subsection{Problem formulation}

This paper employs Modi's model (Modi and Karimi, 1989). It considers the synthesis of $I$ products treated in $J$ batch stages and in $K$ semi-continuous stages. Each batch or semi-continuous stage consists of $m_{j}$ or $n_{k}$ parallel items of same size and type. The item size (continuous variables) and equipments number per stage (discrete variables) are bounded. This model also takes into account short-term storage in intermediate tanks, which divide the whole process into $S$ sub-processes. The economic criterion represents the investment cost for all items :

$$
\operatorname{MinCost}=\sum_{j=1}^{J} a_{j} m_{j} V_{j}^{\alpha_{j}}+\sum_{k=1}^{K} b_{k} n_{k} R_{k}^{\beta_{k}}+\sum_{s=1}^{S-1} c_{s} V_{s}^{\psi_{\gamma_{s}}}
$$

A constraint imposes the $I$ products to be synthesized before a time horizon $H$ :

$$
\sum_{i=1}^{I} H_{i} \leq H
$$

The resulting Mixed Integer Non-Linear Programming (MINLP) problem proves to be non-convex and NP-Hard.

\subsection{Tackled examples}

As above mentioned, a set of increasing complexity examples serves here as bench. This set was built on the basis of two existing problems drawn from the OBPD literature. The first one, consisting of only three batch stages, was proposed by Kocis and Grossmann (1989) for the validation of the Outer Approximation algorithm. The 
second one was presented by Modi and Karimi (1989) and solved with various techniques by various authors (see Section 2.1) : the plant, divided into two subprocesses, consists of four batch stages and six semi-continuous stages. Due to the data similarities between these two problems, four higher size examples were built to have the set complete. Table 1 sums up the studied examples and their respective complexity.

Table 1. Complexity of the studied examples

\begin{tabular}{ccc}
\hline Example & Variables number & Combinatory \\
\hline 1 & 6 & $2.7010^{1}$ \\
2 & 20 & $5.9010^{4}$ \\
3 & 42 & $1.0410^{10}$ \\
4 & 126 & $1.1510^{30}$ \\
5 & 168 & $1.1910^{40}$ \\
6 & 210 & $1.2610^{50}$ \\
\hline
\end{tabular}

Half part of the variables number are discrete. Obviously, the associated combinatory is calculated only according to these discrete variables.

\section{Results and interpretation}

The results for each example, presented in Table 2, were compared in terms of quality and of computational time. Being a stochastic method, the GA was ran 100 times for each example. Its results were analysed according to their repeatability, i.e. their dispersion around the best found solution $F_{G A}{ }^{*}$ (percentage of runs that give a result in the range $\left.\left[F_{G A}{ }^{*}, F_{G A}{ }^{*}+X \%\right]\right)$ and the gap of this latter in comparison with the best found local optimum.

Table 2. Results obtained with DICOPT, SBB and the GA

\begin{tabular}{ccccccc}
\hline Example & 1 & 2 & 3 & 4 & 5 & 6 \\
\hline DICOPT optimum & 166079.2 & 356610.1 & - & - & - & - \\
SBB optimum & 166079.2 & 356610.1 & 957270.7 & 2894504.5 & 3865106.7 & 4859919.7 \\
\hline Best result from GA & 166083.3 & 371771.2 & 982127.4 & 2984418.0 & 3988974.4 & 5000234.6 \\
Gap GA-Optimum (\%) & 0.00 & 4.25 & 2.60 & 3.11 & 3.21 & 4.47 \\
\hline Disp. $\left[F_{G A}{ }^{*}, F_{G A}{ }^{*}+2 \%\right]$ & 99 & 75 & 62 & 36 & 29 & 2 \\
Disp. $\left[F_{G A}{ }^{*}, F_{G A}{ }^{*}+5 \%\right]$ & 100 & 99 & 96 & 59 & 51 & 41 \\
\hline CPU time for DICOPT & $<1 \mathrm{sec}$. & $<1 \mathrm{sec}$. & - & - & - & - \\
CPU time for SBB & $<1 \mathrm{sec}$. & $<1 \mathrm{sec}$. & $4 \mathrm{sec}$. & $3 \mathrm{~min}$. & $29 \mathrm{~min}$. & $7 \mathrm{hrs}$ \\
CPU time for GA & $7 \mathrm{sec}$. & $18 \mathrm{sec}$. & $1 \mathrm{~min}$. & $42 \mathrm{~min}$. & $2.7 \mathrm{hrs}$ & $9.5 \mathrm{hrs}$ \\
\hline
\end{tabular}

The two first examples enable to validate the good behaviour of the GA, in spite of the expectable MP methods superiority concerning the results quality and the CPU time. For example 3, on the one hand, DICOPT has been run for 24 hours and did not find any solution. On the other hand, SBB and the GA keep finding a solution quite efficiently, even if the $2 \%$-dispersion of the GA results decreases of $13 \%$. For that example (and then for the following ones), the constraint handling in GA was carried out with the pseudo-domination method, since the elimination one led to a CPU time upper to 3 hours. 
For examples 4, 5 and 6, despite the exponential CPU time increasing, SBB finds good results. So does the GA, but the CPU time increases a lot, due to the need to use a larger population size to find feasible solutions : the ratio of feasible individuals in the last generation decreases to $37 \%$ in the two last examples, and the number of failures (no feasible individual found during a whole run) reaches $46 \%$ of the runs in example 6 . Nevertheless, this remark underlines the good behaviour of GA when it finds feasible solutions since it counterbalances the $2 \%$ and $5 \%$-dispersion fall. But it also means that a 9.5 hours execution of the GA does not ensure a feasible solution.

\section{Conclusions}

This work presents the application of three optimisation techniques to a set of increasing complexity examples drawn from the OBPD literature. Up to a certain problem size, the based on MP method, DICOPT, failed to give a result in acceptable computational time. But the other MP technique, a Branch and Bound method implemented in the SBB solver solved all the examples, with an increasing but reasonable CPU time. The stochastic technique, a Genetic Algorithm, gave solutions for all examples too. But, in spite of the work made to improve the constraint handling, the difficulty to find a feasible solution seems to make the GA to reach its limits.

Nevertheless, apart from the direct solving efficiency of the Branch and Bound method, the implementation effort has to be taken into account. This MP technique is quite easy to develop, but needs a NLP solver which requires harsh implementation time and experience. Furthermore, from the problem formulation also rise problems of nonconvexities that lead to sub-optimal solutions : these problems can be avoided by a great effort to have the model convex. Experience also shows that an initialisation with the GA results could solve those problems, and lead towards the yet adopted hybridisation technique.

\section{References}

Brooke A., Kendrick D., Meeraus A. and R. Raman, 1998, GAMS User's Guide, GAMS Development Corporation.

Coello Coello C. A. and E. Mezura Montes, 2002, Constraint-handling in genetic algorithms through the use of dominance-based tournament selection, Advanced Engineering Informatics $16,193$.

Duran M. A. and I. E. Grossmann, 1986, An outer-approximation algorithm for a class of mixedinteger nonlinear programs, Mathematical Programming 36, 307.

Kocis G. R. and I. E. Grossmann, 1989, Computational experience with DICOPT solving MINLP problems in process systems engineering, Computer and Chemical Engineering, 13(3), 307.

Modi A. K. and I. A. Karimi, 1989, Design of multiproduct batch processes with finite intermediate storage, Computer and Chemical Engineering, 13, 127.

Patel A. N., Mah R. S. H. and I. A. Karimi, 1991, Preliminary design of multiproduct noncontinuous plants using simulated annealing, Computer and Chemical Engineering, 15, 451.

Pibouleau L., Domenech S., Davin A. and C. Azzaro-Pantel, 2004, Parameterisation of a genetic algorithm, Chemical Engineering Journal, Under Press.

Wang C., Quan H. and X. Xu, 1996, Optimal design of multiproduct batch chemical process using genetic algorithms, Computer and Chemical Engineering, 35, 3560.

Wang C.and Z. Xin, 2002, Ants foraging mechanism in the design of batch chemical process, Computer and Chemical Engineering, 41, 6678. 